\title{
The Impact of Educational Intervention Based on the Theory of Planned Behavior in Choosing Delivery Mode in Primigravida Pregnant Women
}

\author{
Sahar Ghasemi ${ }^{1}$, Mahin Nazari ${ }^{*}$, Homeyra Vafaei ${ }^{2}$, Mohammad Fararouei $^{3}$
}

\begin{abstract}
Objectives: In recent years, despite medical advances, the Cesarean Section (CS) rate and its associated complications remain very high in Iran. This is while the CS carries serious risks for mother and child.

Materials and Methods: This semi-experimental study was carried out on a sample of 87 primigravida women who referred to obstetrics and gynecology clinics affiliated to Shiraz University of Medical Sciences for receiving prenatal care in winter 2014. After a pre-test by using a valid and reliable questionnaire in both groups, educational intervention was provided for the intervention group. The post-test was conducted after one month of the intervention. Then, the data were analyzed by the Statistical Package for the Social Sciences (SPSS) version 20.

Results: The findings showed a significant statistical differences between two groups' behavioral beliefs, outcome evaluation, attitude, control beliefs, perceived power, and perceived behavioral control scores after intervention $(\mathrm{P}<0.05)$.

Conclusion: as the results showed, it is recommend using national health-care policies for planning, implementation and evaluation of training programs based on the theories of behavioral changes, in order to promote the culture of normal vaginal delivery (NVD) and decreasing the rate of CS.

Keywords: Educational intervention, Theory of planned behavior, Delivery mode, Primiparity, Pregnancy
\end{abstract}

\section{Introduction}

Childbirth is a physiological process to end the pregnancy which during it, a child is born. Therefore, the delivery method is extremely important along with it (1). In the meantime, birth of the first child is considered as an important event in the pregnant women's life due to lack of previous experiences. So delivery type is one of the most significant and considerable experiences of the first delivery $(2,3)$. Childbirth is a normal and physiological process which has to happen without any intervention. Despite advances in surgical procedures in recent decades, elective cesarean section (CS) has been increasingly common and raised as a critical issue for healthcare politicians, obstetricians and gynecologists, hospitals as well as mothers (4). The CS refers to the delivery of fetus through incision of abdominal wall and the wall of the uterine (5). The high rate of CS is a modern midwifery problem that raises nowadays, not only enhances obstetric complications, but also imposes an additional financial burden on families, government and insurance institutions (4). The CS rate rises in both developed and developing countries. In a study done by the World Health Organization (WHO) in 2007 and 2008 in 24 countries, the average amount of CS rate was $27 \%$. According to this report, China has the highest rate $(46.2 \%)$. It was also reported that in the United States almost 32\% of deliveries has been through CS. In Iran, several studies have shown that the increasing of CS rate is higher than the recommended rate by the WHO $(10 \%$ $15 \%)$. For example, in Tehran, the CS rate was $66.5 \%$ and it increases to $84 \%$ in the private centers. According to international estimates, the request of mothers has been $4 \%$ to $18 \%$ of all the CSs. In Iran, the rate is variable between $7 \%$ and $13.5 \%(6,7)$. Childbirth through CS is necessary in emergencies and dangerous cases, to save the lives of mother and the child and it worth to accept the dangers of it, but we should not forget that there is no surgery without risk (8). CS has a lot of complications for mother and the child, include bleeding, infection, thromboembolism, increased mortality, premature birth and child breathing problems. CS may increase the risk of problems such as maternal mortality, urinary tract injuries and hysterectomy. On the other hand, mother disabilities after the operation may cause lack of proper breastfeeding and full attention to the child. The results of recent studies show that CS affects the fertility decline and also the creation of postpartum depression $(6,9)$. Overall, the CS, in each country, is one of the performance evaluation indicators for maternal health care programs, and increase in unnecessary CS, indicates inadequate performance of the health care system (10). However, the normal vaginal de-

Received 7 March 2016, Accepted 30 September 2016, Available online 1 January 2017

${ }^{1}$ Department of Health Education and Health Promotion, School of Public Health, Shiraz University of Medical Sciences, Shiraz, Iran. ${ }^{2}$ Maternalfetal Medicine Research Center, Shiraz University of Medical Sciences, Shiraz, Iran. ${ }^{3}$ Department of Epidemiology, School of Public Health, Shiraz University of Medical Sciences, Shiraz, Iran

*Corresponding Author: Mahin Nazari; Tel: (071)37251001; Email: manazari@sums.ac.ir 
livery (NVD) as a normal physiological process has many benefits for both mother and the child (9). For example, it is affordable, the duration of hospital stay after a vaginal birth is less than CS, it does not require anesthesia, and the risk of infection and bleeding afterwards is very low (11).

Some believe that the increase in the CS rate is not only due to medical conditions but it is related to a number of non-medical factors such as: socio-economic and socio-cultural condition, legal and ethical concerns and even cultural and psychological characteristics of women and doctors, too (6). Some studies in Brazil showed that psychosocial factors, such as fear of labor pain, the ability to schedule delivery and physicians' comments are promoters of interventional cultures, has been effective at the high rate of women's request for CS (12). Most primiparous and multiparous believe that CS is safer for the mother and the infant, and NVD may have the risks of prolonged or difficult labor and physical harms. Pelvic floor damage, urinary incontinence, vaginal prolapse and sexual dysfunction which are known as NVD side effects are the other reasons for the request for CS (13). Also, the fear of substandard care during NVD, the effects of childbirth experiences and recommendations of other family members and friends on women's decision-making that believe CS may create a higher level of control over childbirth that leads to security and reduces fear, are the reasons for the preference of CS (14). The study of Abbaspoor et al in Iran showed that women's decision-making is influenced by environmental factors, values, preferences, and a combination of characteristics and personal expectations (6). Pregnant women's preferences are affected by the culture, knowledge about the benefits and risks and social and personal factors. The women's reasons can be classified from perceived easiness of recovery and need to return to family responsibilities to worry about the health of the newborn (15).

Since the behavior of the selection of the type labor is a complex phenomenon and various factors like personal, social and cultural contribute in its development, thus identifying the influencing factors on this behavior can help us to understand this phenomenon better. So that by the exact detection of their decision-making about delivery type, it may lead to the assumptions that according to them and by providing educational programs about CS election, which prevent choosing CS and increase women's desire for NVD (16). The value of health education programs depends on its effectiveness and the effectiveness of health education programs to a large extent depends on the proper use of theories and models used in health education. The theory used in this study is Theory of Planned Behavior (TPB) introduced in 1885 and was developed in 1991 by Ajzen and Fishbin (17). The theory includes the following structures:

Behavior: the way that a person acts. Individual's manner and reactions in different circumstances.

Behavioral intention: the individual's decision to do a behavior.

Attitude: the amount of utility or non-utility of a behav- ior. These two factors lead to its formation: behavioral beliefs and outcome evaluation.

Subjective norms: different people that a person is affected by them and obeys them. Two factors lead to its formation: normative beliefs and motivation for compliance.

Perceived behavioral control: The degree to which an individual feels about to what extent do or not doing a behavior is under his voluntary control. Two factors lead to its formation: control beliefs and perceived power (18).

Since this theory is one of the few models that pays special attention to the individual and social factors of behaviors and can be used in attitude and reproductive behavior. Also, studies based on this theory have shown that educational interventions have been effective in the desired behavior (5). This study aims to investigate the impact of educational intervention on the selection of delivery mode in primigravida pregnant women with the application of the TPB in Shiraz in 2014.

\section{Materials and Methods \\ Study Design}

This is an interventional, semi-experimental study which surveys 87 primigravida women that referred to obstetrics and gynecology clinics of Shahid Ayatollah Motahari and Hafez hospitals affiliated to Shiraz University of Medical Sciences for receiving prenatal care in 2014. These two health centers were selected due to the large number of clientele.

\section{Setting and Sample}

The sample size was estimated that 94 people take place in this research but 87 people remained after deduction of falling (44 participants in interventional group, and 43 in the control group). Sampling was done randomly and within a month. Inclusion criteria included: primigravida pregnant women that for the first time (less than 28 weeks of pregnancy) came to mentioned centers, single fetus pregnancies, lack of indications for CS and any factor that increases the risk of fetal electronic monitoring that can lead to cesarean. Exclusion criteria included absence of more than one session of pregnant women in training sessions, the unavailability of pregnant women in the time of completing the questionnaire, any factor that during the pre-test to post-test, lead to indications for CS.

\section{Measurements}

The data was collecting by researcher-made questionnaire which was about the selection of delivery type. Considering lack of a standard questionnaire in this field, the present questionnaire was made based on the TPB by using resources and textbooks and the results of other studies in this field. The questionnaire includes 80 questions and consists two parts. The first part consisted of demographic questions about the pregnant women and their husbands. This part has 12 questions including age, education, and occupation of pregnant women and their husbands, age of marriage, gestational age (months), type of pregnancy (planned or unplanned), socio-economic class, status of 
health insurance and status of supplementary insurance that was completed, self-report. The second part consists of structures of behavioral beliefs including 15 questions (minimum 15 and maximum 75 points). The outcome evaluation includes 15 questions (minimum 15 and maximum 75 points), with a 5 -point Likert scale (grading 1-5) which collectively constitute the structure of attitude towards the type of delivery (minimum 15 and maximum 375 points). Structure of subjective norms included 7 questions, normative beliefs including 6 questions (minimum 6 and maximum 30 points), with a 5-point Likert scale (grading 1-5) and motivation for compliance with a question. Structures of control beliefs including 15 questions (minimum 15 and maximum 75 points) and perceived power which includes 15 questions (minimum 15 and maximum 75 points), with a 5 -point Likert scale (grading 1-5) which collectively constitute the structure of perceived behavioral control towards the type of delivery (minimum 15 and maximum 375 points). Ultimately structure of behavioral intention included a 4-point Likert scale questions (grading 1-4). The questionnaire was validated by surveying and confirmation of seven of the experts and specialists of health education, epidemiology and psychology. The questionnaire obtained its reliability by using a pilot test and Cronbach a coefficient with these results: behavioral beliefs $0.82 \%$, outcome evaluation $0.78 \%$, normative beliefs $0.67 \%$, control beliefs $0.82 \%$, and perceived power $0.80 \%$.

\section{Data Collection}

The data was collecting by researcher-made questionnaire which was about the selection of delivery type. First, pregnant women completed the pre-test questionnaire. Then, educational interventions were conducted based on the TPB for pregnant women in the intervention group (directly), and their husbands, families and friends (indirectly), while pregnant women in the control group received only routine training by health care staff. Educational interventions were carried out during three 60-minute sessions. The first and second sessions was performed to increase knowledge, change attitudes in field of NVD and CS and their positive assessment of NVD, by training methods of interactive lecture and Q \& A and through providing training booklet and pamphlet. The third meeting was based on perceived behavior control and strengthening pregnant women's intention for NVD. It performed by training methods of interactive lecture, participation and showing educational short videos of the process of NVD and CS. Indirectly educational interventions was carried out during three non-verbal sessions for their subjective norms (husbands, families and friends), by providing the booklet, pamphlet and educational short video. In addition, trainings were followed up by sending text messages. It should be mentioned that there was no possibility to measure the structure of delivery behavior due to time-consuming and non-affordable. The post-test was conducted for a month in the two groups within one month after the completion of educational interventions.
Data Analysis

Data were entered in the Statistical Package for the Social Sciences (SPSS) version 20 and were analyzed by descriptive statistics (mean, standard deviation, frequency, and percentage) and inferential statistics (chi-square, $t$ tests, analysis of covariance [ANCOVA], Spearman correlation coefficient, and nonparametric tests), at.05 significance level.

\section{Results}

The mean age of the pregnant women in the intervention group was $25.52 \pm 4.57$, and it was $25.19 \pm 4.18$ in the control group. The mean age of their husbands in the intervention group was $29.82 \pm 4.77$, and it was $29.37 \pm 3.97$ in the control group. The mean age at marriage among pregnant women in the intervention group was $23.11 \pm 4.41$, and it was $22.72 \pm 4.52$ in the control group. The mean gestational age (months) in the intervention group was $4.59 \pm 1.66$, and it was $4.47 \pm 1.36$ in the control group. Independent-sample $t$ test showed that there is no significant difference in terms of age of pregnant women, age of their husbands, age at marriage and gestational age, between the intervention and control groups $(P>.05)$.

Considering pregnant women's education level, the highest percentage $(45.5 \%)$ was related to diploma degree in the intervention group and the highest percentage $(55.8 \%)$ was related to academic degree in the control group. Regarding husbands, the highest percentage (40.9\%) was related to diploma degree in the intervention group and the highest percentage (37.2\%) was related to diploma and academic degrees in the control group. In term of their occupation, most of the pregnant women were housekeepers in both the intervention group (90.9\%) and the control group (79.1\%). Also, most of their husbands were self-employed in both the intervention group (70.5\%) and the control group (65.1\%). In terms of type of pregnancy, most of the pregnant women reported planned pregnancies both in the intervention group (90.9\%) and the control group (90.7\%). With regards to socioeconomic class, most of the pregnant women were placed in medium level in both the intervention group (59.1\%) and the control group (72.1\%). From the aspect of health insurance, most women had health services insurance in both the intervention group (50.0\%) and the control group (46.5\%). From the aspect of supplementary insurance, most women did not have supplementary insurance both in the intervention group (75.5\%) and the control group $(81.4 \%)$. The chi-square test showed that there is no significant difference between the intervention and control groups, in terms of pregnant women's education level, their husbands' education level, pregnant women's occupation, their husbands' occupation, the type of pregnancy, socioeconomic class, status of health insurance, and status of supplementary insurance $(P>.05)$.

Based on chi-square test, Spearman correlation coefficient and Cramer $\mathrm{V}$ test, there is no significant correlation among any of the socio-demographic variables with the structures of behavioral beliefs, outcome evaluation, 
attitudes, normative beliefs, motivation for compliance, control beliefs, perceived power and perceived behavioral control $(P>.05)$. However, a weak and direct correlation was found between pregnant women's age $(P=.005)$, their husbands' age $(P=.016)$ and marriage age $(P=.019)$, with the structure of behavioral intention. Based on the paired-samples $t$ test, comparing the changes in mean scores of the structures of behavioral beliefs, outcome evaluation, attitudes, normative beliefs, control beliefs, perceived power, and perceived behavioral control in intervention and control groups showed that there is significant difference in the intervention group during the pretest and post-test $(P<.001)$, while in the control group, except the structure of normative beliefs, the mean scores of other structures had no significant differences with each other, before and after the intervention $(P>.05$; Table 1$)$.

Also, according to the Independent-samples $t$ test, comparing the changes in mean scores of structures of behavioral beliefs, outcome evaluation, attitudes, normative beliefs, control beliefs, perceived power, and perceived behavioral control in intervention and control groups, during the pre-test and post-test showed that the intervention and control groups had no significant differences with each other before the intervention $(P>.05)$, while they had significant differences with each other after the intervention, in scores of the structures of behavioral be- liefs $(P<.001)$, outcome evaluation $(P=.005)$, attitude $(P<.001)$, control beliefs $(P=.038)$, and perceived behavioral control ( $P=.013$; Table 1$)$. Well as drawing upon ANCOVA test, a significant difference was observed between the groups in terms of perceived power structure by removing the effect of pre-test $(P<.001)$.

According to the Stuart-Maxwell test, comparing the frequency distribution of motivation for compliance structure in intervention and control groups, during the pre-test and post-test showed that there was no significant difference before and after the intervention both in the intervention group $(P=.274)$ and the control $(P=.183$; Table 2).

Additionally, according to the Stuart-Maxwell test, comparing the frequency distribution of motivation for compliance structure in intervention and control groups, during the pre-test and post-test showed that the intervention and control groups had no a significant difference with each other, before the intervention $(P=.254)$ and after the intervention $(P=.174$; Table 2$)$.

In frequency distribution of motivation for compliance, before and after the educational intervention, pregnant women chose the options of physician, me and my husband in order of priority both, in intervention and control groups (Table 2).

Based on the Wilcoxon test, comparing the frequency

Table 1. Comparing the Changes in Mean Scores of Structures of Behavioral Beliefs, Outcome Evaluation, Attitudes, Normative Beliefs, Control Beliefs, Perceived Power, and Perceived Behavioral Control in Both Intervention and Control Groups, During the Pre-test and Post-test

\begin{tabular}{|c|c|c|c|}
\hline Variables \& Groups & $\begin{array}{c}\text { Pre-test } \\
\text { Mean } \pm \text { SD }\end{array}$ & $\begin{array}{c}\text { Post-test } \\
\text { Mean } \pm \text { SD }\end{array}$ & $\begin{array}{c}P \text { Value } \\
\text { (Paired Samples } T \text { Test) }\end{array}$ \\
\hline \multicolumn{4}{|l|}{ Behavioral beliefs } \\
\hline Intervention group & $49.59 \pm 8.14$ & $55.43 \pm 6.60$ & .000 \\
\hline Control group & $50.21 \pm 6.41$ & $49.02 \pm 6.27$ & .144 \\
\hline$P$ value (independent samples $t$ test) & .695 & .000 & \\
\hline \multicolumn{4}{|l|}{ Outcome evaluation } \\
\hline Intervention group & $46.43 \pm 4.99$ & $51.20 \pm 6.43$ & .000 \\
\hline Control group & $47.37 \pm 5.59$ & $47.58 \pm 5.08$ & .784 \\
\hline$P$ value (independent samples $t$ test) & .410 & .005 & \\
\hline \multicolumn{4}{|l|}{ Attitude } \\
\hline Intervention group & $167.98 \pm 36.65$ & $204.89 \pm 41.52$ & .000 \\
\hline Control group & $172.05 \pm 36.84$ & $170.50 \pm 32.80$ & .708 \\
\hline$P$ value (independent samples $t$ test) & .607 & .000 & \\
\hline \multicolumn{4}{|l|}{ Normative beliefs } \\
\hline Intervention group & $21.91 \pm 3.82$ & $24.70 \pm 3.78$ & .000 \\
\hline Control group & $21.98 \pm 4.45$ & $24.00 \pm 3.04$ & .000 \\
\hline$P$ value (independent samples $t$ test) & .940 & .342 & \\
\hline \multicolumn{4}{|l|}{ Control beliefs } \\
\hline Intervention group & $45.00 \pm 8.71$ & $50.75 \pm 8.26$ & .000 \\
\hline Control group & $47.42 \pm 7.45$ & $47.44 \pm 6.20$ & .978 \\
\hline$P$ value (independent samples $t$ test) & .168 & .038 & \\
\hline \multicolumn{4}{|l|}{ Perceived power } \\
\hline Intervention group & $46.27 \pm 9.90$ & $51.68 \pm 8.30$ & .000 \\
\hline Control group & $49.19 \pm 7.62$ & $49.12 \pm 7.39$ & .922 \\
\hline$P$ value (independent samples $t$ test) & .128 & .132 & \\
\hline \multicolumn{4}{|l|}{ Perceived behavioral control } \\
\hline Intervention group & $156.52 \pm 54.05$ & $194.50 \pm 52.95$ & .000 \\
\hline Control group & $170.91 \pm 44.54$ & $168.98 \pm 39.65$ & .621 \\
\hline$P$ value (independent samples $t$ test) & .180 & .013 & \\
\hline
\end{tabular}


Table 2. Comparing the Frequency Distribution of Motivation for Compliance Structure in Two Intervention and Control Groups, During the Pre-test and Post-test

\begin{tabular}{lccc}
\hline Groups & $\begin{array}{c}\text { Pre-test } \\
\text { No. (\%) }\end{array}$ & $\begin{array}{c}\text { Post-test } \\
\text { No. (\%) }\end{array}$ & $\begin{array}{c}\text { P value (Stuart } \\
\text { Maxwell Test) }\end{array}$ \\
\hline $\begin{array}{l}\text { Intervention group } \\
\text { Physician }\end{array}$ & $27(61.4)$ & $30(68.2)$ & .274 \\
Myself & $8(18.2)$ & $8(18.2)$ & \\
My husband & $8(18.2)$ & $6(13.6)$ & \\
My family & $1(2.3)$ & 0 & \\
Total & $44(100)$ & $44(100)$ & .183 \\
Control group & & & \\
Physician & $20(46.5)$ & $22(51.2)$ & \\
Myself & $14(32.6)$ & $13(30.2)$ & \\
My husband & $9(20.9)$ & $8(18.6)$ & \\
Total & $43(100)$ & $43(100)$ & \\
$P$ value (chi-square test) & .254 & .174 & \\
\hline
\end{tabular}

Table 3. Comparing the Frequency Distribution of Behavioral Intention Structure in Two Intervention and Control Groups, During the Pre-test and Post-test

\begin{tabular}{lccc}
\hline Groups & $\begin{array}{c}\text { Pre-test } \\
\text { No. (\%) }\end{array}$ & $\begin{array}{c}\text { Pre-test } \\
\text { No. (\%) }\end{array}$ & $\begin{array}{c}\text { P Value } \\
\text { (Wilcoxon Test) }\end{array}$ \\
\hline Intervention group & & & .000 \\
Certainly cesarean section & $4(9.1)$ & $2(4.5)$ & \\
Probably cesarean section & $10(22.7)$ & $5(11.4)$ & \\
Probably normal delivery & $25(56.8)$ & $26(59.1)$ & \\
Certainly normal delivery & $5(11.4)$ & $11(25.0)$ & \\
Total & $44(100)$ & $44(100)$ & \\
Control group & & & .157 \\
Certainly cesarean section & $3(7.0)$ & $2(4.7)$ & \\
Probably cesarean section & $9(20.9)$ & $8(18.6)$ & \\
Probably normal delivery & $20(46.5)$ & $21(48.8)$ & \\
Certainly normal delivery & $11(25.6)$ & $12(27.9)$ & \\
Total & $43(100)$ & $43(100)$ & \\
$P$ value (Mann-Whitney test) & .239 & .694 & \\
\hline
\end{tabular}

distribution of behavioral intention structure in two intervention and control groups, during the pre-test and post-test showed that there was significant difference before and after the intervention in the intervention group $(\mathrm{P}<0.001)$, while there was no significant difference in the control group before and after the intervention $(P=.157$;
Table 3).

Likewise according to the Mann-Whitney test, comparing the frequency distribution of behavioral intention structure in intervention and control groups, during the pre-test and the post-test showed that intervention and control groups had no a significant difference with each other before the intervention $(P=.239)$ and after it $(P=.694$; Table 3$)$.

Based on the Spearman correlation coefficient, correlation matrix of behavioral intention structure with the structures of behavioral beliefs, outcome evaluation, attitudes, normative beliefs, control beliefs, perceived power, and perceived behavioral control showed that structures of behavioral beliefs, outcome evaluation, attitude and normative beliefs have direct and medium correlation with behavioral intention structure, while the structures of control beliefs $(r=0.730)$, perceived power $(r=0.761)$ and perceived behavioral control $(r=0.737)$, had a strong and direct correlation with the behavioral intention (Table 4). In addition, Fisher exact test shows that there is no correlation between the structures of motivation for compliance and behavioral intention $(P=.453$; Table 4$)$.

\section{Discussion}

The present study was done using educational intervention based on TPB, and in order to persuade and strengthening primigravida pregnant women's intention for NVD and reduce elective CS in Shiraz, in 2014. Generally the results showed that in the intervention group, the scores of all structures except the structure of motivation for compliance has significant differences with each other before and after the educational intervention. While in the control group except the structure of normative beliefs, the scores of other structures had no significant difference with each other. Moreover, the results showed that the intervention and control groups have no significant difference before educational intervention, while two groups after the intervention, in the scores of structures of behavioral beliefs, outcome evaluation, attitudes, control beliefs, perceived power, and perceived behavioral control had significant differences with each other. This could be due to the fact that the present training program acted more effective than unsystematic and inadequate training in the

Table 4. Spearman Correlation Matrix of Behavioral Intention Structure With Structures of Behavioral Beliefs, Outcome Evaluation, Attitudes, Normative Beliefs, Control Beliefs, Perceived Power, and Perceived Behavioral Control

\begin{tabular}{|c|c|c|c|c|c|c|c|c|}
\hline Variables & $\begin{array}{c}\text { Behavioral } \\
\text { Beliefs }\end{array}$ & $\begin{array}{c}\text { Outcome } \\
\text { Evaluation }\end{array}$ & Attitude & $\begin{array}{c}\text { Normative } \\
\text { Beliefs }\end{array}$ & $\begin{array}{l}\text { Control } \\
\text { Beliefs }\end{array}$ & $\begin{array}{c}\text { Perceived } \\
\text { Power }\end{array}$ & $\begin{array}{c}\text { Perceived Behavioral } \\
\text { Control }\end{array}$ & Intention \\
\hline Behavioral Beliefs & - & $.801 * *$ & $.939 * *$ & $.570 * *$ & $.775 * *$ & $.700 * *$ & $.743 * *$ & $.544 * *$ \\
\hline Outcome Evaluation & $.801 * *$ & - & $.921 * *$ & $.472 * *$ & $.757 * *$ & $.674 * *$ & $.687 * *$ & $.580 * *$ \\
\hline Attitude & $.939 * *$ & $.921 * *$ & - & $.503 * *$ & $.757 * *$ & $.646 * *$ & $.715 * *$ & $.557 * *$ \\
\hline Normative Beliefs & $.570 * *$ & $.472 * *$ & $.503 * *$ & - & $.633 * *$ & $.597 * *$ & $.568 * *$ & $.483 * *$ \\
\hline Control Beliefs & $.775 * *$ & $.757 * *$ & $.757 * *$ & $.633 * *$ & - & $.916 * *$ & $.969 * *$ & $.730 * *$ \\
\hline Perceived Power & $.700 * *$ & $.674 * *$ & $.646 * *$ & $.597 * *$ & $.916 * *$ & - & $.935 * *$ & $.761 * *$ \\
\hline Perceived Behavioral Control & $.743 * *$ & $.687^{* *}$ & $.715^{* *}$ & $.568 * *$ & $.969 * *$ & $.935^{* *}$ & - & $.737 * *$ \\
\hline Intention & $.544 * *$ & $.580 * *$ & $.557 * *$ & $.483 * *$ & $.730 * *$ & $.761 * *$ & $.737 * *$ & - \\
\hline
\end{tabular}


remedial and educational centers. Several studies which applying different training methods have shown the impact of education on strengthening intention and improve the behavioral level of NVD and reduction in CS, but there is no study which investigated all structures of TPB.

The results showed no significant correlation between socio-demographic variables and structures of behavioral beliefs, outcome evaluation, attitudes, normative beliefs, motivation for compliance, control beliefs, perceived power, and perceived behavioral control. But there was a weak and direct correlation between pregnant women's age, their husband's age and their marriage age, with the structure of behavioral intention. The reason which can explain this finding can be small sample size of the study. These findings corresponded with those of the studies by Fathyian et al (19) and Rezakhani Moghaddam et al (20) in the aspects that there was not a significant correlation between pregnant women's education level, occupation and their income with the scores of knowledge, outcome evaluation, attitude, and practice. But they are countercurrent in some points like this: there was no significant correlation between pregnant women's age and mentioned structures. Also in the study conducted by Shahraki Sanavi et al (21) on attitude of pregnant women with intention of elective cesarean based on theory of planned behavior, CS request rate was high in housewives, highly educated women, which is countercurrent with the results of present study (21).

The results showed that in the intervention group after intervention, the mean scores of structures of behavioral beliefs, outcome evaluation and attitude have statistically significant difference in comparison with pre-intervention, while these numbers were not significant in the control group. Also after intervention, the intervention and control groups in terms of the mean scores of mentioned structures had significant differences with each other. In order to justify these findings we can say that behavioral beliefs of people come from the acquisition of information and knowledge. When a person has positive beliefs about the consequences of a behavior, he will have a positive assessment from that behavior and subsequently the person's attitude would be influenced by it (17). This result agreed with the findings of many other studies such as the studies conducted by Sharifirad et al (11), Tofighi Niaki et al (9), Rezakhani Moghaddam et al (20), Besharati et al (17), and Shahraki Sanavi et al (5). For example, the result of the study of Besharati et al on the effect of educational interventions based on TPB in selecting delivery mode among pregnant women showed significant differences between intervention and control groups in the mean scores of knowledge, outcome evaluation, and attitude among pregnant women after the educational intervention.

The results showed that after the intervention, the mean score of normative beliefs structure have significant differences compared with the two groups before the intervention, but the groups have no significant difference with each other after the intervention. After the intervention also, the frequency distribution of motivation for compli- ance structure had no statistically significant differences compared with the pre-intervention and also compared with each other in the intervention and control groups. This could be due to the fact that subjective norms, received trainings indirectly and by the pregnant women. Also subjective norms are formed gradually and along the time. Thus, short-term training cannot be very effective on them. In the countercurrent conducted by Caron et al (22) on evaluation of a theoretically based AIDS/STD peer education program on postponing sexual intercourse and on condom use among adolescents attending high school, the findings showed that those who were in the experimental group positively modified their personal normative beliefs compared with the control group. Also in the other countercurrent study conducted by Besharati et al (17) on the effect of educational interventions based on TPB in selecting delivery mode among pregnant women a significant difference was found between pregnant women in both groups in the mean score of subjective norms after the intervention.

In frequency distribution of motivation for compliance, before and after the educational intervention, pregnant women chose the options of physician, me and my husband in order of priority both, in intervention and control groups. In the interpreting this finding we can say that physician's opinion as a specialist and valid person, and her husband's beliefs as the closest person in relation to pregnant women are very important for them. Furthermore, the effect of physician-oriented norms in society is undeniable (20). This finding corresponded with the results of study of Shahraki Sanavi et al (5). In the study of Fathyian et al (19) and Sharifirad et al (11), motivation to compliance from the physician was in the first place, their husband was in the second place, their mother was in the third place, and friends, books, the mass media and health care staff were in the next rankings, respectively.

The results showed that after the educational intervention, the mean scores of structures of control beliefs, perceived power, and perceived behavioral control have statistically significant difference in comparison with pre-intervention, while these numbers were not significant in the control group. Also after intervention, the intervention and control groups in terms of the mean scores of mentioned structures had significant differences with each other. It can also be attributed to the fact that the intervention group received the educational program in comparison with the control group. These findings corresponded with the results of many other studies. For example, in the study of Shahraki Sanavi et al (5) on the effect of education on base the TPB toward normal delivery in pregnant women with intention elective cesarean, a significant difference was observed in the scores of perceived behavioral control among pregnant women in the intervention and the control group after the educational intervention. The findings of the study by Caron et al (22)on postponing sexual intercourse and on condom use among adolescents attending high school were inconsistent with those of the present study, showing a significant positive 
modification of perceived behavioral control in order to postponing sexual intercourse (22).

The results showed that after the educational intervention, the frequency distribution of behavioral intention structure in the intervention group has statistically significant difference compared with pre-intervention, while this difference was not significant in the control group. After the educational intervention also, the two groups did not show statistically significant difference with each other in terms of the frequency distribution of behavioral intention. This finding can also be justified considering the fact that the intention to NVD improved as a result of educational intervention in the intervention group, but not enough to create a statistically significant difference between the intervention and control groups. Compared to pregnant women in the control group, those in the intervention group significantly modified their intention for NVD after the intervention. These findings corresponded with those of the studies of Fathyian et al (19), Besharati et al (17), and Shahraki Sanavi et al (5). Peyman and Samiee Roudi's research results on education based on the TPB on caries prevention of permanent teeth showed a significant difference before and after the educational intervention so this finding is not in consistent with the present study (23). Also the findings of the study by Casper (24) on the theory of planned behavior applied to continuing education for mental health professionals showed that guiding the class by the theory of planned behavior significantly and substantially increases the participants' intentions to use the self-report tool that assesses felt need for employment among people with serious mental illnesses in comparison with the standard classes (24).

The results showed that structures of control beliefs, perceived power, and perceived behavioral control have a stronger relationship with intention of delivery behavior. This might be attributed to the fact that this three structures refers to person's beliefs and perception of their own ability to do a behavior and that doing a stressful behavior such as delivery can be how much easy or difficult for pregnant women (18). Certainly in the case of delivery behavior, these structures have greater stability and strength. In this field a perfectly consonant study was not found. But in the study of Shahraki Sanavi et al (5) it was found that the most predictor of behavior are two structures of behavioral intention and perceived behavioral control.

\section{Conclusion}

The results showed that training the primigravida pregnant women using the TPB has been effective in the promotion of information, attitudes, perceived behavioral control and also to encourage and strengthen the intention of pregnant women for NVD. Therefore, it is recommended to adapt comprehensive systematic policies of national health care in order to planning, implementation and evaluation of consultative-educational programs based on theories of behavior change to persuade the pregnant women to accept NVD and prevent the propagation of CS culture. Also, by paying attention to this fact that the most women's motivation for compliance is their physician and her/his recommendations, it is necessary to apply a suitable strategy introduced by the health-care politicians in order to justify obstetricians and gynecologists to improve the level of NVD and reduce the rate of CS.

\section{Conflict of Interests}

There is no conflict of interest with the authors.

\section{Ethical Issues}

This research has the permission of Shiraz University of Medical Sciences and Research Vice-chancellor of the University, and also the approval of Ethics Committee of the University (No. 93-7214), with IRCTID: IRCT201411287816N3. For this purpose, the voluntary informed consent was obtained from all participants. After getting the necessary information about how the study will be implemented and its duration, they signed informed consent form voluntarily, and became aware that their information will remain confidentially. In addition, participants are excluded from the study, anytime they were unwilling.

\section{Financial Support}

The study has been financially supported by Shiraz University of Medical Sciences

\section{Acknowledgments}

Sahar Ghasemi has written this article for her MSc thesis in Health Education (No. 93-7214) Shiraz University of Medical Sciences. Hereby, the authors would like to thank the Research Vice-chancellor of the University and School of Health for their cooperation. They are also grateful to Ms S. Sayyadi for improving the use of English in the manuscript. We also want to thanks all the staff of remedial and educational centers of Shahid Motahari and Hafez and all the pregnant women who helped me to complete this research project.

\section{References}

1. Eynsheykh ZD, Shaahmadi F, Taslimi S, Emamiureh F, Moeinaldini $S$. Investigating the relationship between demographic factors and choice of delivery method in pregnant women in the city of Savojbolagh. J Fam Reprod Health. 2013;7(1):35.

2. Toosi M, Akbarzadeh M, Zare N, Sharif F. Effect of attachment training on anxiety and attachment behaviors of first-time mothers (Persian). Hayat. 2011;17(3):69-79.

3. Guittier M-J, Cedraschi C, Jamei N, Boulvain M, Guillemin F. Impact of mode of delivery on the birth experience in first-time mothers: a qualitative study. BMC Pregnancy Childbirth. 2014;14:254. doi:10.1186/1471-2393-14-254.

4. Sanavi FS, Rakhshani F, Ansari-Moghaddam A, Edalatian $\mathrm{M}$. Reasons for elective cesarean section amongst pregnant women; a qualitative study. J Reprod Infertil. 2012;13(4):237.

5. Shahraki Sanavi F, Navidian A, Rakhshani F, Ansarimoghadam A. The effect of education on base the theory of planned behavior toward normal delivery in pregnant women with intention elective cesarean (Persian). 
Hormozgan Medical Journal. 2012;17(6):531-9.

6. Abbaspoor Z, Moghaddam-Banaem L, Ahmadi F, Kazemnejad A. Iranian mothers' selection of a birth method in the context of perceived norms: a content analysis study. Midwifery. 2014;30(7):804-809. doi:10.1016/j. midw.2013.06.003.

7. Hajian S, Shariati M, Mirzaei Najmabadi Kh, Yunesian M, Ajami ME. Psychological predictors of intention to deliver vaginally through the extended parallel process model: a mixed-method approach in pregnant iranian women. Oman Med. 2013;28(6):395-403. doi: 10.5001/ omj.2013.115.

8. Mohammaditabar SH, Kiani A, Heidari M. The survey on tendencies of primiparous women for selecting the mode of delivery (Persian). Journal of Babol University of Medical Sciences. 2009;11(3):54-59.

9. Tofighi Niaki M, Behmanesh F, Mashmuli F, Azimi H. The effect of prenatal group education on knowledge, attitude and selection of delivery type in primiparous women. Iran J Med Educ. 2010;10(2):124-130.

10. Poorhidary M, Sozani A, Kasaeian A. The survey of knowledge and attitude of pregnant women referred to Medical Centers of Qom towards type of delivery (Persian). Journal of Knowledge \& Health. 2007;2(2):28-34.

11. Sharifirad G, Rezaeian M, Soltani R, Javaheri S, Amidi Mazaheri M. A survey on the effects of husbands education of pregnant women on knowledge, attitude and reducing elective cesarean section. J Health Syst Res. 2010;6(1):7-13.

12. Yazdizadeh B, Nedjat S, Mohammad K, Rashidian A, Changizi N, Majdzadeh R. Cesarean section rate in Iran, multidimensional approaches for behavioral change of providers: a qualitative study. BMC Health Serv Res. 2011;11:159. doi:10.1186/1472-6963-11-159.

13. Faisal I, Matinnia N, Hejar AR, Khodakarami Z. Why do primigravidae request caesarean section in a normal pregnancy? A qualitative study in Iran. Midwifery. 2013;30(2):227-233. doi:10.1016/j.midw.2013.08.011.

14. Fenwick J, Staff L, Gamble J, Creedy DK, Bayes S. Why do women request caesarean section in a normal, healthy first pregnancy? Midwifery. 2010;26(4):394-400. doi:10.1016/j. midw.2008.10.011.

15. Liu NH, Mazzoni A, Zamberlin N, et al. Preferences for mode of delivery in nulliparous Argentinean women: a qualitative study. Reprod Health. 2013;10(1):2. doi:10.1186/1742-4755-10-2.

16. Nourani Saadodin S, Goudarzi M, Peyman N, Esmaily H.
Prediction of intrauterine device insertion among women of childbearing age based on the theory of planned behavior (Persian). The Iranian Journal of Obstetrics, Gynecology and Infertility. 2013;16(45):13-19.

17. Besharati F, Hazavehei S, Moeini B, Moghimbeigi A. Effect of educational interventions based on theory of planned behavior (TPB) in selecting delivery mode among pregnant women referred to Rasht health centers (Persian). Zahedan Journal of Research in Medical Sciences. 2011;19(77):94106.

18. Saffari M, Shojaeizadeh D, Ghofranipour F, Heidarnia AR, Pakpour Hajyagha A. Theory of reasoned action and theory of planned behavior. Theories, models and methods of health education and health promotion (In Persian). Tehran: Asar Sobhan; 2012:78.

19. Fathyian Z, Sharifirad G, Hassanzadeh M. The effect of education on khowledge, attitude and function in pregnant women than normal vaginal delivery, according behavioral intention model in Khomainy-Shahr. J Tabib Shargh. 2007;9(2):123-31.

20. Rezakhani Moghaddam H, Shojaeizadeh D, Taghdisi MH, Hamidzadeh Arbabi Y, Savadpour MT. The effect of education by community health volunteers on choice of delivery kind in pregnant women based on the behavioral intention model (BIM. Journal of School of Public Health and Institute of Public Health Research. 2013;10(3):27-40.

21. Shahraki-Sanavi F, Rakhshani F, Navidiyan A, AnsariMoghaddam A. A Study on attitude of pregnant women with intention of elective cesarean based on theory of planned behavior (Persian). Zahedan Journal of Research in Medical Sciences. 2012;14(9):95-7.

22. Caron F, Godin G, Otis J, Lambert LD. Evaluation of a theoretically based AIDS/STD peer education program on postponing sexual intercourse and on condom use among adolescents attending high school. Health Educ Res. 2004;19(2):185-197. doi:10.1093/her/cyg017.

23. Peyman N, Samiee Roudi K. The effect of education based on the theory of planned behavior on caries prevention of permanent teeth in fifth grade students in Khaf City. Journal of Mashhad Dental School. 2015;39(2):123-36. doi: 10.22038/jmds.2015.4065.

24. Casper ES. The theory of planned behavior applied to continuing education for mental health professionals. Psychiatric Services. 2007;58(10):1324-1329. doi:10.1176/ ps.2007.58.10.1324.

Copyright (C) 2017 The Author(s); This is an open-access article distributed under the terms of the Creative Commons Attribution License (http://creativecommons.org/licenses/by/4.0), which permits unrestricted use, distribution, and reproduction in any medium, provided the original work is properly cited. 